\title{
Lumbar Spinal Steroid Injections and Infection Risk after Spinal Surgery: A Systematic Review and Meta-Analysis
}

\author{
Harshadkumar A. Patel ${ }^{1}$, Naga Suresh Cheppalli ${ }^{2}$, Amit Wasudeo Bhandarkar ${ }^{3}$, Vidhi Patel ${ }^{4}$, Anuj Singla ${ }^{5}$ \\ ${ }^{1}$ Department of Orthopaedic Surgery, Westchester Medical Center, Valhalla, NY, USA \\ ${ }^{2}$ Department of Orthopaedics and Rehabilitation, University of New Mexico, VA Hospital, Albuquerque, NM, USA \\ ${ }^{3}$ Department of Orthopaedics, SSM Health St Mary's Hospital, Centralia, IL, USA \\ ${ }^{4}$ Zilmed Inc., Marietta, GA, USA \\ ${ }^{5}$ Department of Orthopaedics, University of Virginia Health System, Charlottesville, VA, USA
}

Lumbar spinal steroid injections (LSSI) are universally used as preferred diagnostic or therapeutic treatment options before major spinal surgeries. Some recent studies have reported higher risks of surgical-site infection (SSI) for spinal surgeries performed after injections, while others have overlooked such associations. The purpose of this study is to systematically review the literature and perform a meta-analysis to evaluate the associations between preoperative LSSI and postoperative infection following subsequent lumbar decompression and fusion procedures. Three databases, namely PubMed, Scopus, and Cochrane Library, were searched for relevant studies that reported the association of spinal surgery SSI with spinal injections. After the comprehensive sequential screening of the titles, abstracts, and full articles, nine studies were included in a systematic review, and eight studies were included in the meta-analysis. Studies were critically appraised for bias using the validated MINOR (methodological index for non-randomized studies) score. The odds ratio (OR) and 95\% confidence interval (CI) were calculated. Subgroup analysis was performed according to the time between LSSI and surgery and the type of lumbar spine surgery. Meta-analysis showed that preoperative LSSI within 30 days of lumbar spine surgery was associated with significantly higher postoperative infection compared with the control group (OR,1.79; 95\% $\mathrm{Cl}, 1.08-2.96)$. Based on subgroup analysis, lumbar spine fusion surgery within 30 days of preoperative LSSI was associated with significantly high-infection rates (OR, 2.67; 95\% Cl, 2.12-3.35), while no association was found between preoperative LSSI and postoperative infection for lumbar spine decompression surgeries. In summary, given the absence of high-level studies in the literature, careful clinical interpretation of the results should be performed. The overall risk of SSI was slightly higher if the spinal surgery was performed within 30 days after LSSIs. The risk was higher for lumbar fusion cases but not for decompression-only procedures.

Keywords: Lumbosacral region; Spinal injections; Lumbar spine surgery; Surgical wound infection; Systematic review

\section{Introduction}

Lumbar spinal steroid injections (LSSIs) are the most common invasive nonsurgical interventions performed to alleviate lower back pain or radiculopathy caused by disc herniations, degenerative spine disease, and/or lumbar

Received May 2, 2021; Revised Jul 10, 2021; Accepted Jul 27, 2021

Corresponding author: Harshadkumar A. Patel

Department of Orthopaedic Surgery, Westchester Medical Center, 100 Woods Road, Valhalla, NY 10595, USA

Tel: +1-914-789-2700, Fax: +1-914-789-2745, E-mail: harshadkumar.patel@wmchealth.org 
canal stenosis [1]. A steep rise in the use of LSSI has been documented among the Medicare population since the 2000s [2]. It is the preferred initial treatment for most spinal degenerative and stenotic pathologies as a diagnostic and prognostic adjunct and in some cases as a definitive treatment [3-5]. LSSI is directed to either epidural interlaminar, epidural transforaminal, or facet joints, depending on the spinal pathology and pain pattern.

LSSI works by reducing nerve root edema and local ischemia and inhibiting the pre-inflammatory cytokines through its anti-inflammatory mechanism [6]. Even though LSSI are generally considered a safer intervention, there are reported risks of vasovagal episodes, pain exacerbation, injection site soreness, and headaches [7]. Apart from these risks, the recent literature has expressed some concern that immunomodulatory and anti-inflammatory effects of the steroid can alter the local host environment, making it more susceptible to postoperative infection after subsequent lumbar spine surgeries. Several retrospective and database studies have reported an increased postoperative infection rate in patients who have received preoperative LSSI [8-11], while other studies have concluded no association [12-15]. Given the potentially devastating consequences associated with spinal surgical-site infections, it is of paramount importance to determine whether such a relationship truly exists or not. Timing of the LSSI from the index surgery and the type of the lumbar spine surgery, either decompression surgery or lumbar instrumented fusion surgery, are the other factors that also influence the association of LSSI and postoperative infection $[9,12]$. Considering the highly variable spinal pathologies and their severity, variable injection/surgical techniques, and very low rates of postoperative spine infections, it would be difficult to conduct multicenter prospective or randomized control trials with sufficient study power to evaluate the association of injections and infection risk. In this scenario, systematic data pooling and meta-analysis of the non-randomized studies of interventions could provide robust and much-desired evidence [16].

The purpose of this study was to systematically review the literature and meta-analyze the impact of the preoperative LSSI on postoperative infection in lumbar spine surgery. The study's primary aim was to find the association between preoperative LSSI timing and postoperative infection after lumbar spinal surgery. The secondary aim was to stratify the association according to the lumbar spine surgery decompression vs. fusion surgery type.

\section{Materials and Methods}

A systematic review was performed according to the preferred reporting items for systematic reviews and metaanalysis (PRISMA) guidance [17]. Inclusion criteria were set as follows: Studies must have (1) included the comparative cohorts of the study group ofpatients who have received preoperative lumbar corticosteroid injections, and the control group of patients without preoperative lumbar epidural steroid injection; (2) shown the temporal time association of LSSI to the index lumbar spinal surgery; (3) reported postoperative outcomes, such as infection, seroma, and/or return to the operating room for incision and debridement for surgical wounds; and (4) reported postoperative follow-up data for at least 30 days.

The studies which met the following criteria were excluded: (1) non-English language publications; (2) studies published in low-tier journals; (3) studies with cohorts of less than 10 patients were excluded given the very low rate of postoperative infection after spinal surgery; and (4) case reports, reviews, editorials, or technique papers.

A systematic search of the PubMed, Scopus, and Cochrane Library databases was conducted from the year of inception until January 2021 using the following keywords/phrases: [corticosteroid injection OR epidural injection OR steroid injection OR epidural steroid injection] AND [spine surgery OR lumbar spine surgery] AND [infection OR postoperative complication], without adding any filters. Two independent authors (H.A.P. and N.S.C.) performed the study selection and data extraction. The study titles and abstracts were reviewed to determine the study eligibility. After the exclusion of nonrelevant papers, full texts of the remaining studies were reviewed to assess eligibility. Finally, the included studies' references were also reviewed manually to identify the additional studies for inclusion. Any discrepancies for the selected studies were settled by the consensus between investigators with the help of a third author (A.S). Included studies were appraised critically using the validated version of the methodological index for non-randomized studies (MINORs) and scored out of 24 points [18]. Whenever there was a lack of information noted in studies, the studies' authors were contacted to gather additional information.

The study's primary outcome was the postoperative infection within 90 days of the index lumbar spine surgery. The data were systematically stratified into three groups to check the temporal effect of the LSSI: (1) LSSI within 30 
days of index procedure (LSSI <30), (2) LSSI within 30-90 days of index surgery (LSSI 30-90), and (3) LSSI more than 90 days before the index surgery (LSSI >90). Whenever a study had divided their patients into more than three groups, data were pooled together $[13,15]$. Some database studies have used matched control groups, which can have overlapping patient populations among different control groups $[11,19]$. In these particular studies, data pooling from different study groups was avoided.

A meta-analysis was performed according to the time of LSSI from the index surgery and type of the lumbar surgery reported in the study, decompression $[8,11,12,15]$, fusion $[9,12,13,20]$, or combined [14,19]. The software Review Manager ver. 5.4.1 (RevMan, Copenhagen, Denmark) was used to conduct statistical analyses. A randomeffect analysis model was used in conjunction with the Mantel-Haenszel statistical method. Summary odds ratio (OR)s and 95\% confidence interval (CI)s were calculated and reported for the outcome for each study. Heterogeneity was assessed using $\operatorname{tau}^{2}, I^{2}, \mathrm{Q}$, and $p$-values.

\section{Results}

The literature search based on three databases identified 715 articles, of which 415 were left after duplication removal. Nine studies met the inclusion criteria after following the PRISMA protocol, as shown in Fig. 1 [8,9,11$15,19,20]$. Two studies have used the same database with overlapping years $[8,11]$. Thus, only one of those studies was used for meta-analysis to avoid overlapping the patient population [11]. Nine studies were used for systematic qualitative review $[8,9,11-15,19,20]$, and eight studies were used for quantitative meta-analysis $[9,11-15,19,20]$ (Tables 1,2).

Meta-analysis showed that preoperative LSSI within 30 days of index lumbar spine surgery was associated with significantly higher postoperative infection compared with the control group, while no association was identified for preoperative LSSI $>30$ days before surgery (LSSI $<30$ : OR, 1.79; 95\% CI, 1.08-2.96; $p=0.02$; heterogenicity: $\operatorname{tau}^{2}=0.40, I^{2}=90 \%$; LSSI 30-90: OR, 1.17; 95\% CI, 0.84-1.63; $p=0.34$; heterogenicity: $\operatorname{tau}^{2}=0.10, I^{2}=64 \%$; LSSI $>90$ : OR, 1.01; 95\% CI, 0.86-1.18; $p=0.90$; heterogenicity: $\operatorname{tau}^{2}=0.01, I^{2}=40 \%$ ) (Figs. $2-4$ ). On subgroup analysis, lumbar spine fusion surgery within 30 days of preoperative LSSI was found to be associated with a significantly high-infection rate (LSSI <30: fusion surgery: OR, 2.67;
95\% CI, 2.12-3.35; $p \leq 0.01$; heterogenicity: $\operatorname{tau}^{2}=0.00$, $I^{2}=0 \%$ ) (Fig. 1). No association was found between preoperative LSSI and postoperative infection for lumbar spine decompression surgery (Figs. 1-3). The overall infection rate ranged from $0 \%$ to $10.48 \%$ among different studies (Table 2). The pooled infection rate for patients with preoperative LSSI $<30$ days was $2.18 \%$ versus $1.40 \%$ in control group, whereas for LSSI (30-90 days) it was 1.63\% versus $1.33 \%$ for the control group.

Table 3 reports a critical appraisal of the included studies using MINORS criteria. One study was a prospective cohort study [20], two studies were retrospective comparative cohort studies $[12,14]$, while the remaining six studies were retrospective database studies $[9,11,13-15,19]$. A clearly stated aim was overserved in all the studies. No study has reported unbiased assessments of outcomes.

\section{Discussion}

LSSI have shown good-to-excellent effectiveness in treating radiculitis secondary to disc herniations and fair effectiveness in treating radiculitis, secondary to spinal stenosis and axial back pain [21]. Patients' perceptions of the spinal conditions and spinal surgery, the relative safety of the LSSI, and the patient's and surgeon's desires to exhaust all the conservative options before proceeding with the surgery are a few of the reasons for the consistent rise in the use of LSSI [22-24]. A recent study of 52,935 patients who received LSSI reported an extremely low $(0.011 \%)$ rate of major complications [25]. There has been a growing interest in the spine community to evaluate the impact of these relatively safe injections on subsequent spinal surgeries. Studies have shown the possible risk of infections in patients undergoing lumbar spine surgery after the injection $[8-11,26]$. During this controversy, patients and surgeons both need to know what implications these benign injections can have if surgery is undertaken soon.

Our meta-analysis has found a small, but significant, increased rate of infection in the LSSI group compared with the control group following lumbar spine surgery, combined decompression, and/or fusion surgery if the LSSI was administered within 30 days (OR, 1.79; 95\% CI, 1.082.96). This association was noted to be stronger for fusion surgery (OR, 2.67; 95\% CI, 2.12-3.35) and nonsignificant for decompression surgery (OR, 2.04; 95\% CI, 0.96-4.34) on subgroup analysis. Similar results have been reported by Singla et al. [9] (OR, 2.59; 95\% CI, 2.01-3.34), Kreitz 


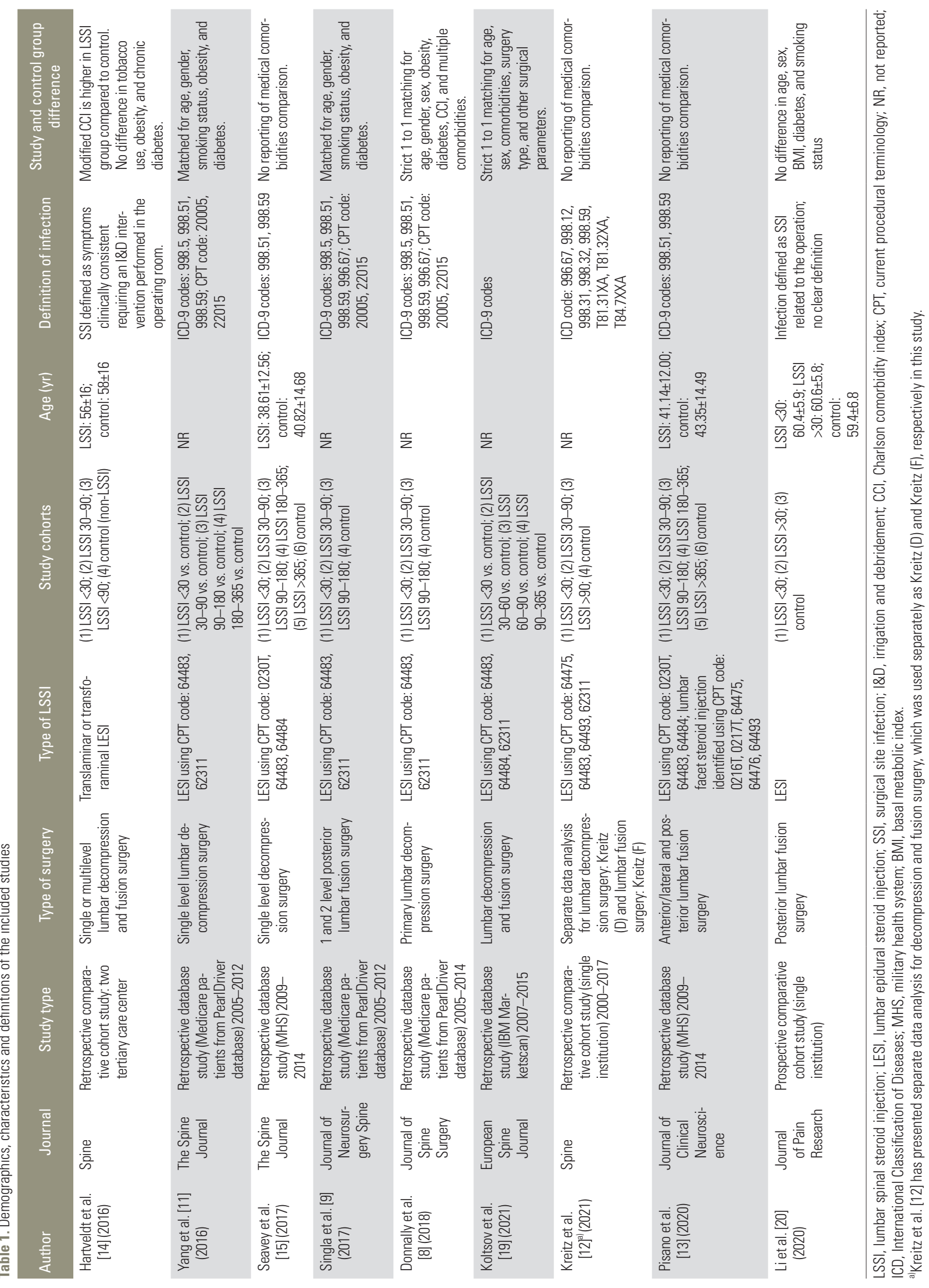




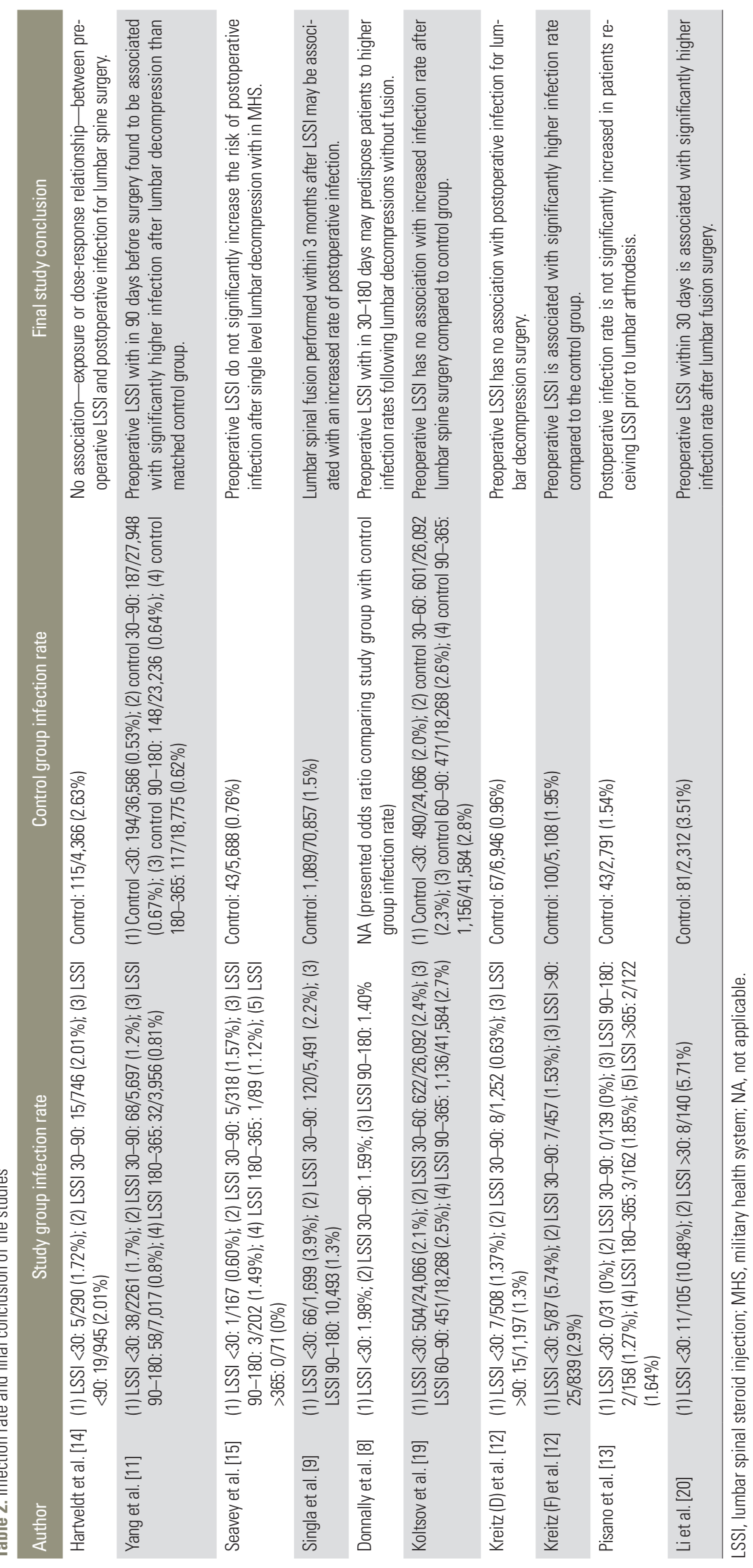


et al. [12] (OR, 3.05; 95\% CI, 1.21-7.70), and Li et al. [20] (OR, 3.22; 95\% CI, 1.66-6.25) for fusion surgery if patients

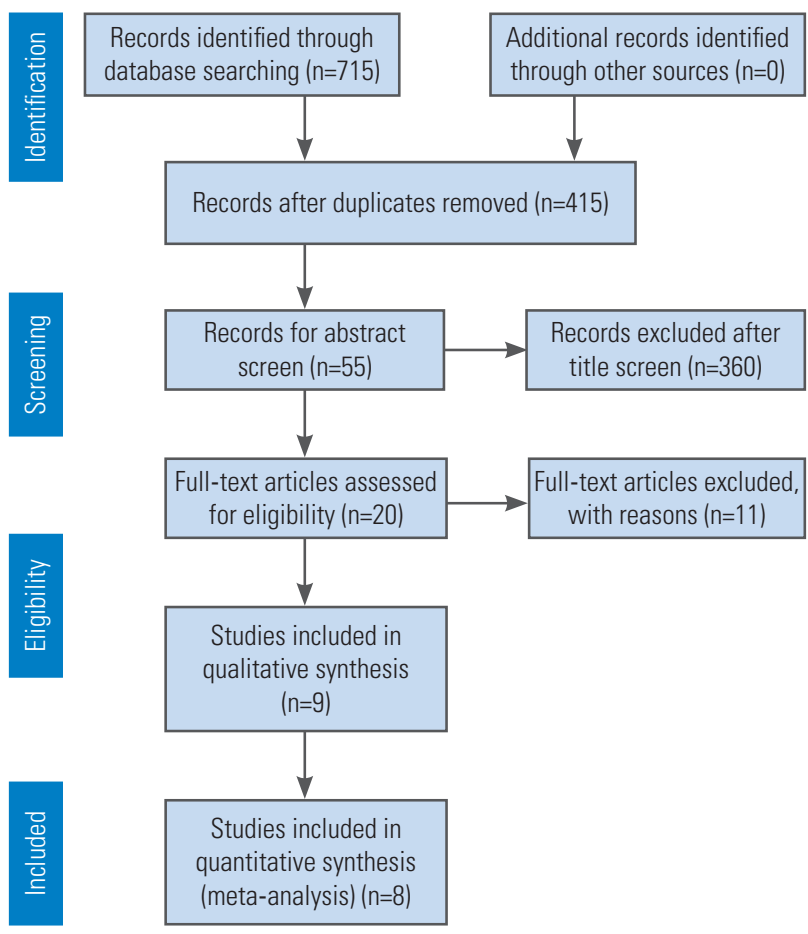

Fig. 1. PRISMA (preferred reporting items for systematic reviews and metaanalysis) flowchart showing literature search and methodology of selection. had received LSSI within 30 days. However, meta-analysis has tightened the $\mathrm{CI}$ for this association (OR, 2.67; 95\% CI, 2.12-3.35). Our meta-analysis has refuted the association between preoperative LSSI within 30-90 days and postoperative infection, which was reported by individual studies, such as Donnally et al. [8] (OR, 4.69; 95\% CI, 2.44-9.02), Yang et al. [11] (OR, 1.79; 95\% CI, 1.36-2.37), and Singla et al. [9] (OR, 1.43; 95\% CI, 1.18-1.73). A large database study by Yang et al. [11] has also shown significant association with nearly three times higher odds (OR, 3.21; 95\% CI, 2.26-4.55) of infection if the patients had received LSSI within 30 days of the lumbar decompression surgery. This association became nonsignificant as the data were pooled from multiple similar studies, showing the effect of the increased study power in meta-analysis.

LSSI can directly inoculate bacteria/organisms from skin flora, especially when the proper sterile precautions have not been taken $[6,27]$. Locally injected steroids have antiinflammatory and immunomodulatory actions. These actions compromise the local environment and limit the immunological response to the indolent or early infection if it occurs [6]. There is evidence of systemic cortisol suppression at 3 weeks following LSSI with long-acting corticosteroid formulations, thus suggesting sustained systemic absorption of the corticosteroid $[28,29]$. The elimination

Table 3. Critical appraisal of the studies using MINORS criteria

\begin{tabular}{|c|c|c|c|c|c|c|c|c|c|}
\hline Criteria & $\begin{array}{l}\text { Hartveldt et } \\
\text { al. [14] }\end{array}$ & $\begin{array}{l}\text { Yang et al. } \\
\text { [11] }\end{array}$ & $\begin{array}{l}\text { Seavey et al. } \\
\text { [15] }\end{array}$ & $\begin{array}{l}\text { Singla et al. } \\
{[9]}\end{array}$ & $\begin{array}{c}\text { Donnally et al. } \\
{[8]}\end{array}$ & $\begin{array}{l}\text { Koltsov et al. } \\
\text { [19] }\end{array}$ & $\begin{array}{l}\text { Kreitz et al. } \\
\text { [12] }\end{array}$ & $\begin{array}{l}\text { Pisano et al. } \\
\text { [13] }\end{array}$ & $\begin{array}{l}\text { Li et al. } \\
\text { [20] }\end{array}$ \\
\hline A clearly stated aim & 2 & 2 & 2 & 2 & 2 & 2 & 2 & 2 & 2 \\
\hline Inclusion of consecutive patients & 1 & 2 & 2 & 2 & 2 & 2 & 2 & 2 & 2 \\
\hline Prospective collection of data & 0 & 0 & 0 & 0 & 0 & 0 & 0 & 0 & 2 \\
\hline $\begin{array}{l}\text { Endpoints appropriate to the aim of } \\
\text { the study }\end{array}$ & 2 & 2 & 2 & 2 & 2 & 2 & 2 & 2 & 2 \\
\hline $\begin{array}{l}\text { Unbiased assessment of the study } \\
\text { endpoint }\end{array}$ & 0 & 0 & 0 & 0 & 0 & 0 & 0 & 0 & 0 \\
\hline $\begin{array}{l}\text { Follow-up period appropriate to the } \\
\text { aim of the study }\end{array}$ & 2 & 2 & 2 & 2 & 2 & 2 & 2 & 2 & 2 \\
\hline Loss to follow up less than $5 \%$ & 2 & 2 & 2 & 2 & 2 & 2 & 2 & 2 & 2 \\
\hline $\begin{array}{l}\text { Prospective calculation of the study } \\
\text { size }\end{array}$ & 0 & 0 & 0 & 0 & 0 & 0 & 0 & 0 & 0 \\
\hline An adequate control group & 2 & 2 & 2 & 2 & 2 & 2 & 2 & 2 & 2 \\
\hline Contemporary groups & 2 & 2 & 2 & 2 & 2 & 2 & 2 & 2 & 2 \\
\hline Baseline equivalence of groups & 1 & 2 & 0 & 2 & 2 & 0 & 0 & 0 & 2 \\
\hline Adequate statistical analyses & 2 & 2 & 2 & 2 & 2 & 1 & 2 & 2 & 2 \\
\hline Total & 16 & 18 & 16 & 18 & 18 & 15 & 16 & 16 & 20 \\
\hline
\end{tabular}

The items are scored 0 (not reported), 1 (reported but inadequate), or 2 (reported and adequate). The global ideal score being 16 for non-comparative studies and 24 for comparative studies.

MINORS, methodological index for non-randomized studies. 


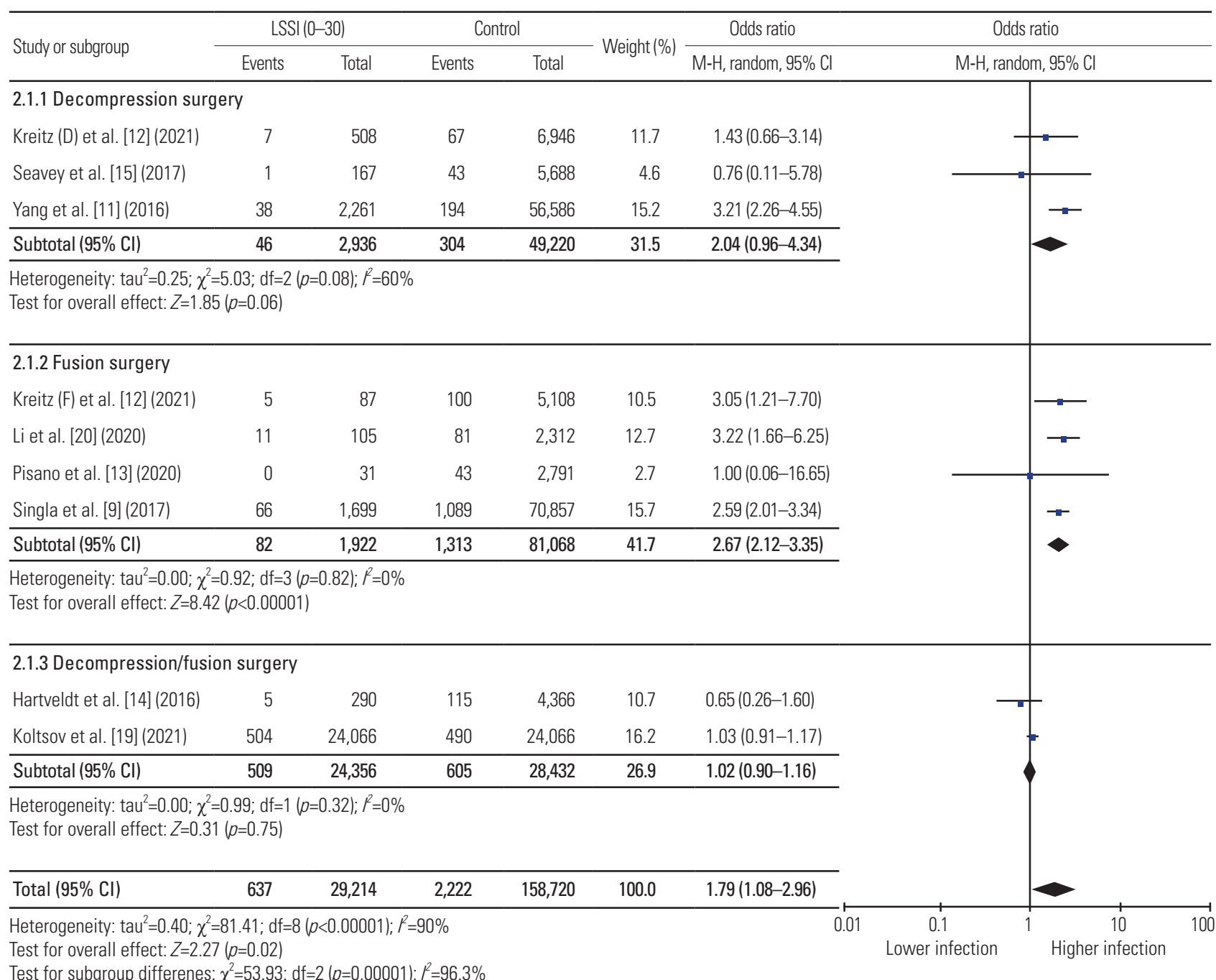

Fig. 2. Results of meta-analysis for preoperative lumbar spinal steroid injection (LSSI) with in 30 days of index lumbar spine surgery. Cl, confidence interval; df, degrees of freedom.

of half-life of particulate steroids like triamcinolone is approximately 3 weeks [18]. Even though the exact pharmacokinetics of these theories have not been explained, one can reasonably assume that these processes can be locally active for weeks, if not months, considering that the patients who receive these injections report relief of symptoms for many months [9]. This may be a reason for the transition of infection association from significant to nonsignificant depending on the timing of injection in relation to the index surgery. This may also support our result of the declining infection rate from $2.18 \%$ in the group where LSSI $<30$ days to $1.64 \%$ in the group with LSSI: 30-90 days. Patients with severe stenosis and severe symptoms are less likely to benefit from the injection and may opt for surgery soon after the injection. Severe stenosis itself can be a risk factor for longer operation times and thus for higher infection rates. Thus, a positive association between infection and LSSI within 30 days may be attributed to the severity of pathology instead of LSSI or to both.

The intraoperative epidural steroid is routinely used following discectomy/decompression surgery to reduce short-term postoperative pain, narcotics requirements, and epidural fibrosis [30,31]. If preoperative LSSI affects the local environment by sustained immunomodulatory and anti-inflammatory effects, intraoperative steroids would have the same effect. Furthermore, contrary to expectations, a meta-analysis of the 17 studies has found that intraoperative epidural steroids are not associated with postoperative infection following discectomy (risk ratio, 4.58; 95\% CI, 0.72-5.26) [31]. These findings are in line with our 


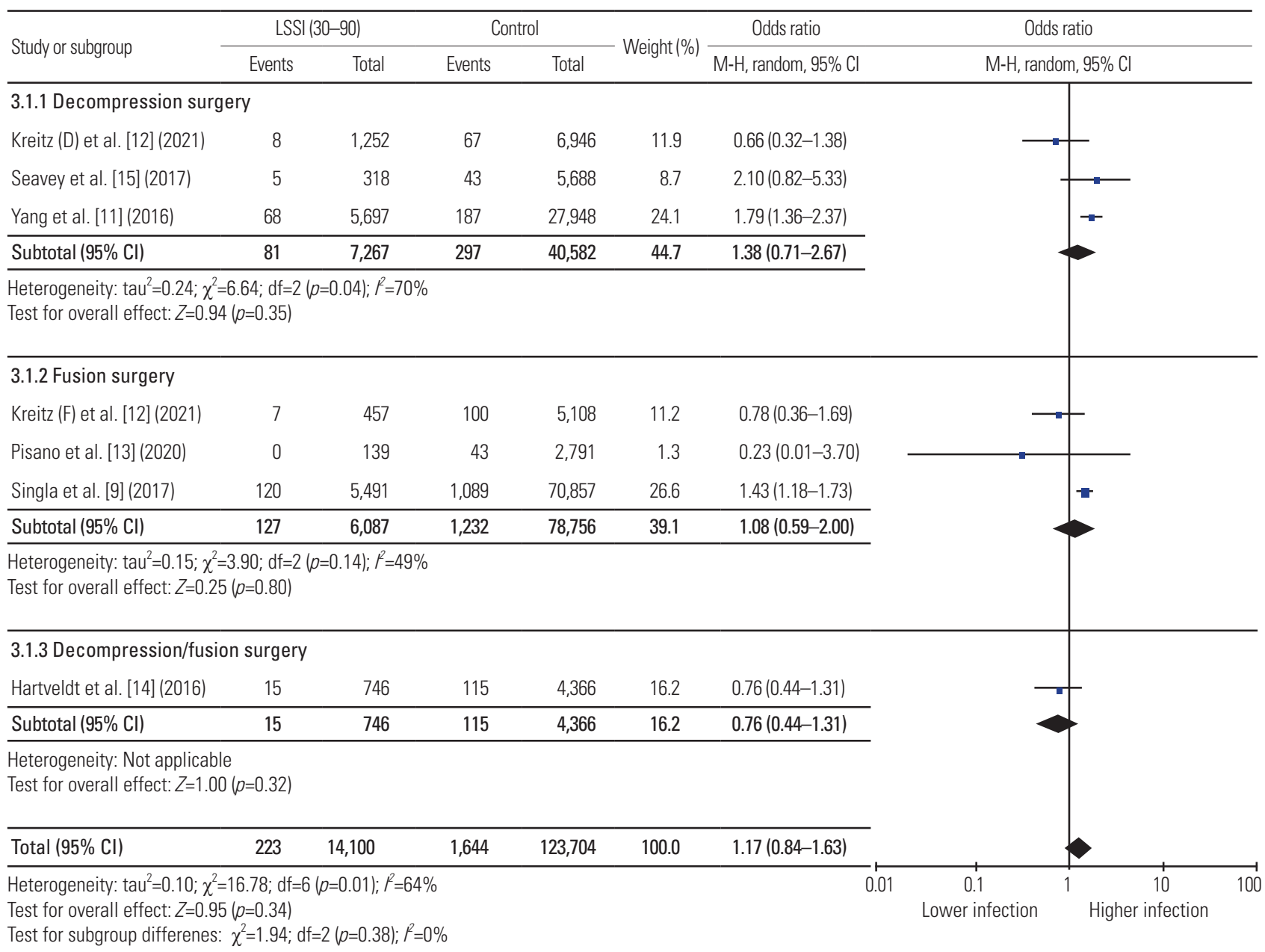

Fig. 3. Results of meta-analysis for preoperative lumbar spinal steroid injection (LSSI) between 30-90 days of index lumbar spine surgery. Cl, confidence interval; df, degrees of freedom.

results of non-association between preoperative LSSI and postoperative infection following decompression surgery (OR, 2.04; 95\% CI, 0.96-4.34). Decompression surgeries are inherently smaller procedures with less blood loss, less operating time, and less invasiveness compared to fusion surgeries; thus, they are associated with a relatively smaller risk for infections than fusion surgery. Additionally, the presence of synthetic bone grafts and hardware, that is, foreign bodies, may further increase the risk of infection, which may explain the significant association of LSSI with postoperative infection following fusion surgery (OR, 2.67; 95\% CI, 2.12-3.35) compared with decompression surgery.

Even though specific studies have shown similar, mixed results for intra-articular injections and infection rates in subsequent total-hip and knee-joint arthroplasties, metaanalysis of the comparative studies has refuted the association with postoperative superficial infection (risk ratio,
1.75; 95\% CI, 0.76-4.04) and deep-tissue infection (risk ratio, 1.87; 95\% CI, 0.80-4.35) [32]. In contrast, our study has consistently found the association between steroid injection and fusion surgeries. After controlling for the patient-specific variables, broad surgical technique variations exist in lumbar spine surgery compared with total joint arthroplasty, which inherently affects the outcome of the pooled data across different studies, especially database studies.

The main limitation of the current review is the heterogeneity with some aspects of the included studies. Broad variation in the infection rate has been reported. For example, the infection rate of $10.48 \%$ for LSSI $<30$ days was reported by Li et al. [20], whereas Pisano et al. [13] reported that it was $0.00 \%$. These findings are suggestive of some hidden heterogeneity among the different study populations. There was no standardized definition of the 


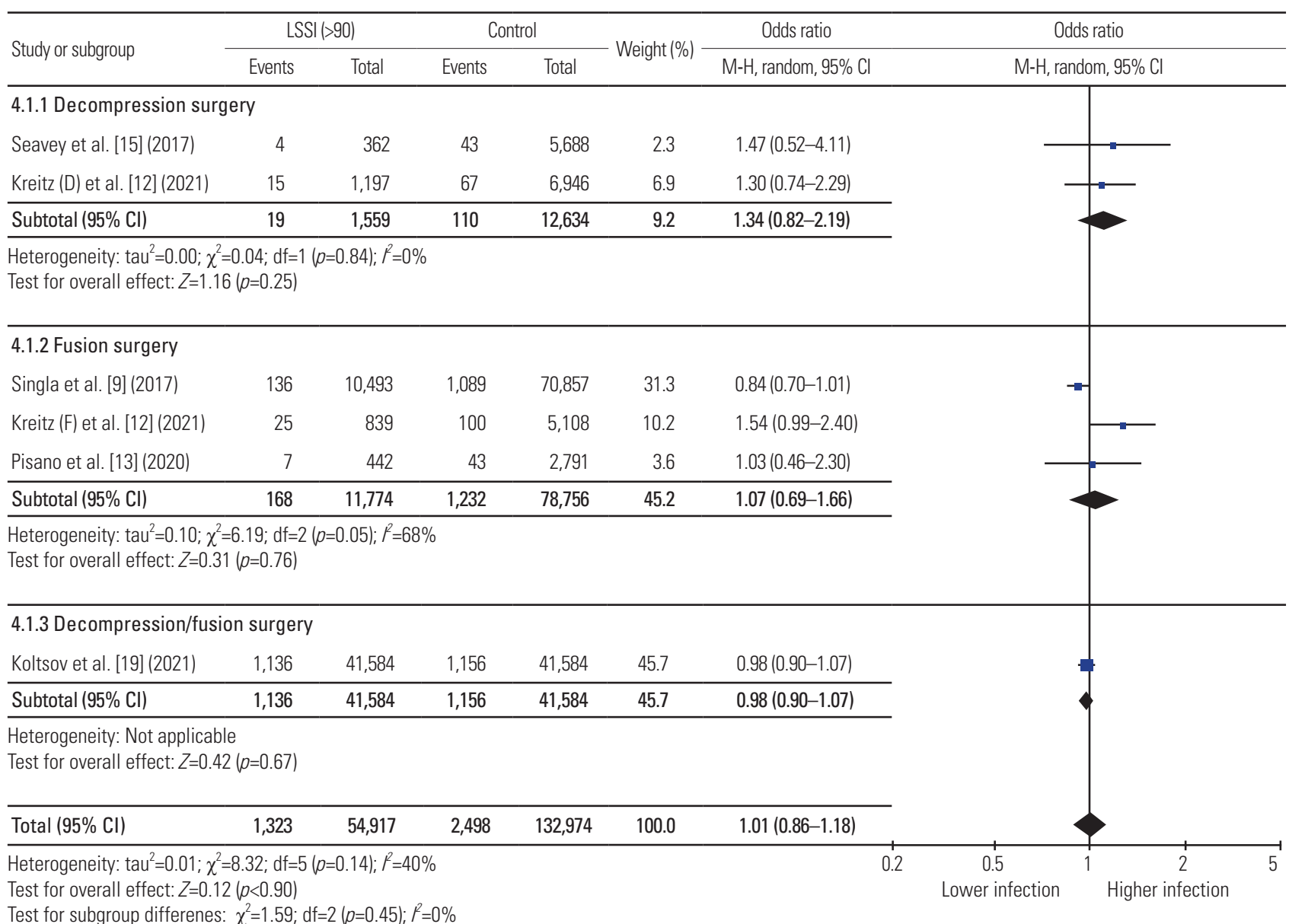

Fig. 4. Results of meta-analysis for preoperative lumbar spinal steroid injection (LSSI) beyond 90 days of index lumbar spine surgery. Cl, confidence interval; df, degrees of freedom.

infection across all the studies (Table 1). Some studies have not matched their study and control groups for confounder variables for infection, including demographic, surgical factors (minimally invasive surgery versus open surgery, anterior versus lateral versus posterior fusion), and patient-related factors, including age, sex, body mass index, diabetes, smoking status, operating time, and Charlson comorbidity index $[12,13,15]$. A broad variation exists for the age distribution for the included studies. Specifically, some studies focused only on the Medicare population $[8,9,11]$, while others have included all age patients $[12,13,15]$. All studies have reported LSSI as a dichotomous variable (the event occurred: yes/no) and the time of the event. The number of LSSI, particulate versus nonparticulate steroids, type of LSSI, clinic versus operating room LSSI, post-injection antibiotics use, adjunctive use of systematic steroids, the complexity of the surgical procedure, use of intraoperative osteosynthesis material, such as bone graft, are unknown because of the database limitations. Except for one [20], all other studies have limitations attributed to the retrospective nature of the study design. There were six database studies, which rely on data retrieval through the current procedural terminology (CPT) and International Classification of Diseases (ICD) codes $[8,9,11,13,15,19]$. Thus, the coding error at data entry can affect the study population and (subsequently) the study outcome. Apart from this, minor variations have been noticed for CPT and ICD codes across all the database studies (Table 1). Because of the overlapping control group, data pooling was avoided in the metaanalyses of the studies of Koltsov et al. [19] for the group with LSSI: 30-90 days, and the study of Yang et al. [11] for the group with LSSI $>90$ days, which can affect the overall meta-analysis outcome for these groups. The study's main 
strength is a robust patient population from pooled studies that helped the rejection of the association of LSSI and infection for decompression surgery and further affirmed the association between LSSI and infection with a narrow CI for fusion surgery.

\section{Conclusions}

The overall postoperative infection rate remains very low despite the use of the preoperative LSSI. Our results suggest that preoperative LSSI within 30 days before the lumbar fusion surgery is associated with a small, yet a statistically significant, increased infection rate. There is no association between preoperative LSSI and postoperative infection following lumbar decompression surgery. Given the limitation of the available literature, the meta-analysis result needs to be interpreted carefully for clinical applications. Additional large-sized cohort studies conducted based on a standardized injection protocol and infection definition would be valuable to examine whether preoperative LSSI close to the lumbar fusion surgery is safe or not.

\section{Conflict of Interest}

No potential conflict of interest relevant to this article was reported.

\section{Acknowledgments}

We would like to thank you Mark Cote, PhD. Biostatistician, director of outcomes, research and quality, Department of Orthopedic Surgery at University of Connecticut for data analysis guidance.

\section{Author Contributions}

Each author has significantly contributed to the study. HAP and NSC are involved in study, design, data collection and manuscript preparation. VP contributed in data analysis and manuscript preparation. AWB and AS contributed by expert opinion and in manuscript preparation.

\section{References}

1. Epstein NE. The risks of epidural and transforaminal steroid injections in the spine: commentary and a comprehensive review of the literature. Surg Neurol Int 2013;4(Suppl 2):S74-93.
2. Manchikanti L, Pampati V, Boswell MV, Smith HS, Hirsch JA. Analysis of the growth of epidural injections and costs in the Medicare population: a comparative evaluation of 1997, 2002, and 2006 data. Pain Physician 2010;13:199-212.

3. Kaufmann TJ, Geske JR, Murthy NS, et al. Clinical effectiveness of single lumbar transforaminal epidural steroid injections. Pain Med 2013;14:1126-33.

4. Bhatia A, Flamer D, Shah PS, Cohen SP. Transforaminal epidural steroid injections for treating lumbosacral radicular pain from herniated intervertebral discs: a systematic review and meta-analysis. Anesth Analg 2016;122:857-70.

5. Manson NA, McKeon MD, Abraham EP. Transforaminal epidural steroid injections prevent the need for surgery in patients with sciatica secondary to lumbar disc herniation: a retrospective case series. Can J Surg 2013;56:89-96.

6. McLain RF, Kapural L, Mekhail NA. Epidural steroid therapy for back and leg pain: mechanisms of action and efficacy. Spine J 2005;5:191-201.

7. Plastaras C, McCormick ZL, Garvan C, et al. Adverse events associated with fluoroscopically guided lumbosacral transforaminal epidural steroid injections. Spine J 2015;15:2157-65.

8. Donnally CJ 3rd, Rush AJ 3rd, Rivera S, et al. An epidural steroid injection in the 6 months preceding a lumbar decompression without fusion predisposes patients to post-operative infections. J Spine Surg 2018;4:529-33.

9. Singla A, Yang S, Werner BC, et al. The impact of preoperative epidural injections on postoperative infection in lumbar fusion surgery. J Neurosurg Spine 2017;26:645-9.

10. Zusman N, Munch JL, Ching A, Hart R, Yoo J. Preoperative epidural spinal injections increase the risk of surgical wound complications but do not affect overall complication risk or patient-perceived outcomes. J Neurosurg Spine 2015;23:652-5.

11. Yang S, Werner BC, Cancienne JM, et al. Preoperative epidural injections are associated with increased risk of infection after single-level lumbar decompression. Spine J 2016;16:191-6.

12. Kreitz TM, Mangan J, Schroeder GD, et al. Do preoperative epidural steroid injections increase the risk of infection after lumbar spine surgery? Spine (Phila Pa 1976) 2021;46:E197-202. 
13. Pisano AJ, Seavey JG, Steelman TJ, Fredericks DR Jr, Helgeson MD, Wagner SC. The effect of lumbar corticosteroid injections on postoperative infection in lumbar arthrodesis surgery. J Clin Neurosci 2020;71:66-9.

14. Hartveldt S, Janssen SJ, Wood KB, et al. Is there an association of epidural corticosteroid injection with postoperative surgical site infection after surgery for lumbar degenerative spine disease? Spine (Phila $\mathrm{Pa}$ 1976) 2016;41:1542-7.

15. Seavey JG, Balazs GC, Steelman T, Helgeson M, Gwinn DE, Wagner SC. The effect of preoperative lumbar epidural corticosteroid injection on postoperative infection rate in patients undergoing singlelevel lumbar decompression. Spine J 2017;17:120914.

16. Reeves BC, Deeks JJ, Higgins JP, Shea B, Tugwell P, Wells GA. Chapter 24: Including non-randomized studies on intervention effects. In: Higgins JP, Thomas J, Chandler J, et al., editors. Cochrane handbook for systematic reviews of interventions, version 6.1 (updated September 2020) [Internet]. London: Cochrane; 2020 [cited 2021 May 1]. Available from: https://training.cochrane.org/handbook/current/ chapter-24.

17. Moher D, Liberati A, Tetzlaff J, Altman DG; PRISMA Group. Preferred reporting items for systematic reviews and meta-analyses: the PRISMA statement. BMJ 2009;339:b2535.

18. Slim K, Nini E, Forestier D, Kwiatkowski F, Panis Y, Chipponi J. Methodological index for non-randomized studies (MINORS): development and validation of a new instrument. ANZ J Surg 2003;73:712-6.

19. Koltsov J, Smuck MW, Alamin TF, Wood KB, Cheng I, Hu SS. Preoperative epidural steroid injections are not associated with increased rates of infection and dural tear in lumbar spine surgery. Eur Spine J 2021;30:870-7.

20. Li P, Hou X, Gao L, Zheng X. Infection risk of lumbar epidural injection in the operating theatre prior to lumbar fusion surgery. J Pain Res 2020;13:2181-6.

21. Benyamin RM, Manchikanti L, Parr AT, et al. The effectiveness of lumbar interlaminar epidural injections in managing chronic low back and lower extremity pain. Pain Physician 2012;15:E363-404.
22. Radcliff KE, Rihn J, Hilibrand A, et al. Does the duration of symptoms in patients with spinal stenosis and degenerative spondylolisthesis affect outcomes?: analysis of the Spine Outcomes Research Trial. Spine (Phila Pa 1976) 2011;36:2197-210.

23. Weinstein JN, Tosteson TD, Lurie JD, et al. Surgical versus nonoperative treatment for lumbar spinal stenosis four-year results of the Spine Patient Outcomes Research Trial. Spine (Phila Pa 1976) 2010;35:132938.

24. Friedly JL, Bresnahan BW, Comstock B, et al. Study protocol: lumbar epidural steroid injections for spinal stenosis (LESS): a double-blind randomized controlled trial of epidural steroid injections for lumbar spinal stenosis among older adults. BMC Musculoskelet Disord 2012;13:48.

25. Lee JW, Lee E, Lee GY, Kang Y, Ahn JM, Kang HS. Epidural steroid injection-related events requiring hospitalisation or emergency room visits among 52,935 procedures performed at a single centre. Eur Radiol 2018;28:418-27.

26. Labaran LA, Puvanesarajah V, Rao SS, et al. Recent preoperative lumbar epidural steroid injection is an independent risk factor for incidental durotomy during lumbar discectomy. Global Spine J 2019;9:807-12.

27. Kraeutler MJ, Bozzay JD, Walker MP, John K. Spinal subdural abscess following epidural steroid injection. J Neurosurg Spine 2015;22:90-3.

28. Friedly JL, Comstock BA, Turner JA, et al. A randomized trial of epidural glucocorticoid injections for spinal stenosis. N Engl J Med 2014;371:11-21.

29. Friedly JL, Comstock BA, Heagerty PJ, et al. Systemic effects of epidural steroid injections for spinal stenosis. Pain 2018;159:876-83.

30. Jamjoom BA, Jamjoom AB. Efficacy of intraoperative epidural steroids in lumbar discectomy: a systematic review. BMC Musculoskelet Disord 2014;15:146.

31. Akinduro OO, Miller BA, Haussen DC, Pradilla G, Ahmad FU. Complications of intraoperative epidural steroid use in lumbar discectomy: a systematic review and meta-analysis. Neurosurg Focus 2015;39:E12.

32. Charalambous CP, Prodromidis AD, Kwaees TA. Do intra-articular steroid injections increase infection rates in subsequent arthroplasty?: a systematic review and meta-analysis of comparative studies. J Arthroplasty 2014;29:2175-80. 